
\title{
R Reserach S Suare \\ Geriatric scores as single-handed tools in the risk of 30-day mortality after hip fracture
}

\section{Carmen da Casa}

Instituto de Investigacion Biomedica de Salamanca

\section{Carmen Pablos-Hernández}

Hospital Universitario de Salamanca

Alfonso González-Ramírez

Hospital Universitario de Salamanca

Juan F Blanco ( $\square$ jfblanco@usal.es )

Hospital Universitario de Salamanca https://orcid.org/0000-0001-7803-9149

\section{Research article}

Keywords: Comprehensive Geriatric Assessment, hip fracture, mortality, geriatric scores

Posted Date: July 30th, 2020

DOI: https://doi.org/10.21203/rs.3.rs-48407/v1

License: (9) This work is licensed under a Creative Commons Attribution 4.0 International License. Read Full License 


\section{Abstract \\ Background}

The 30-day mortality rate after hip fracture surgery has been considered as an indirect indicator of the quality of care. This work aims to validate the Barthel Index, Katz Index, Lawton-Brody Index, and Physical Red Cross Scale analyzed in the Comprehensive Geriatric Assessment at admission as predictors of 30-day mortality after hip fracture surgery.

\section{Methods}

Prospective study including 899 hip fracture patients over 65. Bed-ridden, non- surgically treated patients, and high energy trauma or tumoral etiology fractures were excluded. Variables distribution were assessed by $\chi^{2}$, and we performed binary logistic regression for risk assessment. $P<0.05$ was considered statistically significant.

\section{Results}

We noted a 30-day mortality rate of $5.9 \%$. We related Barthel Index $(O R=0.986[0.975-0.996], p=0.010)$, Katz Index (OR per point $=1.254$ [1.089-1.444], $p=0.002$ ), Lawton-Brody Index (OR $=0.885$ [0.7880.992], $p=0.037)$, and Physical Red Cross Scale ( $O R=1.483$ [1.094-2.011], $p=0.011)$ with the 30-day mortality of patients after hip fracture surgery. We also validate the Barthel Index inflection point (0-55) $\left(\mathrm{OR}_{\mathrm{BI}(0-55)}=2.428\right.$ [1.379-4.275], $\left.\mathrm{p}=0.002\right)$ and Katz Index inflection point $(\mathrm{A}-\mathrm{B})\left(\mathrm{OR}_{\mathrm{KI}(\mathrm{A}-\mathrm{B})}=0.493\right.$ [0.273-0.891], $p=0.019)$ for the assessment of the highest risked patients.

\section{Conclusions}

The geriatric scores would be useful multifunctional tools in the assessment of hip fracture patients as singly predictors of 30-day mortality.

\section{Background}

One of the most interesting topics in hip fracture management is to clarify indicators to foresee the results of the surgical treatment of this complex group of patients. Once known, these indicators would allow us to establish preventive measures and allocate resources for those patients with a higher risk of mortality.

The treatment of older hip fracture patients requires a multidisciplinary approach carried out in the socalled Orthogeriatric Units. The Comprehensive Geriatric Assessment (CGA) is a key tool throughout the patient admission process reporting on the functional situation of the patients that has been related to 
the hip fracture outcome (1). The CGA includes the evaluation of some geriatric scores and geriatric syndromes $(2,3)$. Barthel Index (BI), Katz Index (KI), and Lawton-Brody Index (LBI) scores are broadly used in a standardized way as part of the CGA. The Physical Red Cross Scale (PCRS) is also a mobility score widely used in Spain.

These geriatric scores, along with clinical information of the patient, elucidate the functional situation of the patient and provides a deeper insight on patients which would help in in-hospital monitoring and later follow-up.

We dispose of a variety of tools to predict the outcome of surgical management of hip fracture patients such as the NHFS, AHFS, or O-Possum $(4,5)$, although, given the variability between the world population and health-care models, their application could be compromised by the availability of certain resources. Some geriatric scores have been also previously studied as predictors of in-hospital mortality for patients undergoing surgery (6). Our working group demonstrated the usefulness of geriatric scores as predictors of long-term mortality in a limited number of patients (7), being able to establish geriatric score inflection points from which the risk of long-term survival was significantly decreased.

The aim of this work is to validate $\mathrm{BI}, \mathrm{Ki}, \mathrm{LBI}$, and PRCS as predictors of early mortality in older hip fracture patients. We also aim to establish the possible usefulness of the geriatric scores inflection points previously defined on $\mathrm{BI}, \mathrm{KI}$, and $\mathrm{LBI}$ on the 30 -day mortality rate in older hip fracture patients.

\section{Methods}

\section{Design and population}

We designed a prospective observational study, including all patients 65-years-old and older with hip fracture admitted from January 12018 to December 312019 in the orthogeriatric unit of the University Hospital of Salamanca (UHS). We excluded bed-ridden patients (PRCS $=5$ ) and those cases that were not surgically treated, as well as all those patients who suffered fractures due to high energy trauma or who showed tumoral etiology. All patients included in the study expressed their consent to participate by signed consent.

Overall, a complete CGA was performed in 919 patients during the study period. We follow-up patients for 30 -day mortality, assuming the attrition rate of $2.2 \%$, leading to a cohort of 899 patients undergoing surgery after hip fracture.

Demographic and clinical variables

On admission, sociodemographic variables such as gender, date of birth and admission, and place of residence were collected. We also recorded the type of fracture, the type of surgical procedure, and destination at discharge.

Geriatric Scores 
In the UHS Orthogeriatric Unit, the following geriatric scores are used as part of CGA: Barthel Index, Katz Index, Lawton-Brody Index and Physical Red Cross Scale.

The $\mathrm{BI}(8)$ collects data on the degree of capability for the development of 10 basic activities of daily living (ADL). For each activity analyzed, a gradual score is applied in 5 points, according to the patient's ability to perform it. BI punctuations go from 0 to 100.

The KI (9) estimates the independence of the patient to perform basic ADL. It analyses 6 functions from which categorization of patients originates, possible situations ranging from the total independence named with the letter $A$ to the total dependence indicated with the letter $G$.

The LBI (10) evaluates instrumental activities of daily living (IADL). The LBI assigns a score from 0 to 8; the highest score indicates the best functional capability. Due to the characteristics of the IADL analyzed, LBI has been traditionally applied differentiating the gender of the patient and limiting to 4 the maximum score for men (11). At UHS, the whole questionnaire was determined regardless of patient gender.

For these geriatric scores, we have previously described an inflection point from which the survival rate of patients after hip fracture surgery would be significantly abridged (7). The inflection points were determined by the largest mean difference in survival tendency.

The Physical Red Cross Scale (12) evaluates the physical ambulatory ability for the patient, similar to the Functional Ambulation Classification (FAC). The physical-status evaluation of PRCS is concerning 5 levels of ambulatory ability from 0 , which indicates full capability, to 5 , which indicates any ambulatory capability. This score was also used as an exclusion variable in our study for bed-ridden patients (PCRS = $5)$.

\section{Statistical analysis}

Data were imported into a database for statistical analysis with the IBM ${ }^{\circledR}$ SPSS $®$ Statistics program (v.25). Descriptive statistics included mean and standard deviation, and normality of sample distribution was defined by the Kolmogorov-Smirnoff test. We ascertain the statistically significant differences among groups by $\chi^{2}$. The Odds Ratio (OR) was estimated by binary logistic regression. In all cases, a $\mathrm{p}$ value of less than 0.05 was considered statistically significant.

\section{Results}

The study population was composed of 899 patients. One in four patients were men and the mean age was greater than 85 years old (Table 1). We noted a 30-day mortality rate of $5.9 \%$. Figure 1 shows the early mortality rates represented over the population on each category of the four geriatric scores analyzed. 
We addressed the place of residence of patients, noting that $51.6 \%$ of patients were residents from municipalities with more than 12,500 inhabitants and $32.0 \%$ of participants were living on an older people specific healthcare institution before admission. A total of 472 patients were institutionalized at discharge $-32.8 \%$ of non-institutionalized patients at admission were institutionalized at discharge - . A social worker was involved in $17.6 \%$ of cases. Incidence by type of hip fracture was also studied, showing that most cases it was a trochanteric fracture, whose surgical intervention treatment was osteosynthesisbased (Table 1).

The mean time to surgery was $2.94 \pm 2.55$ days, noting $34.1 \%$ of patients operated within the first $48 \mathrm{~h}$, and $17.7 \%$ of patients operated within the first $24 \mathrm{~h}$.

Barthel Index.

The mean punctuation in $\mathrm{BI}$ of our population was $72.8 \pm 23.44$. We noted significant decreasing $\mathrm{BI}$ on aging groups $(p<0.001)$, and lower $B$ I punctuation on women $(p=0.013)$ and institutionalized patients at admission $(p<0.001)$, but no differences on BI were achieved for new institutionalized patients at discharge $(p<0.05)$, or regarding the time to surgery of patients $(<24 h / 48 h, p>0.05)$.

The distributions of BI punctuation regarding the 30-day mortality of patients are shown in Table 2 and represented in Figure 2. BI was significantly related to 30-day mortality (OR per point=0.986 [0.975-0.996], $\mathrm{p}=0.010)$.

The inflection point previously determined for BI (BIIP) was set at 60 points, grouping patients presenting moderate and severe dependence (BI 0-55) and patients showing slight dependence or total independence (IB 60-100). Analyzing the study population by the distribution of BIIP, we observe no differences regarding the gender of patients, new institutionalized patients at discharge or time to surgery $(p>0.05$, in all cases), but with institutionalized patients at admission $(p<0.001)$ and aging patients $(p<0.001)$. We noted a significantly increased risk for early mortality at this point, noting that moderate or severe dependent patients had double increased risk $\left(\mathrm{OR}_{\mathrm{BI}(0-55)}=2.428\right.$ [1.379-4.275], $\left.\mathrm{p}=0.002\right)$.

\section{Katz Index}

The most frequent category of $\mathrm{KI}$ on our population was $\mathrm{A}(31.9 \%)$. We noted significant decreasing $\mathrm{KI}$ on aging groups $(p<0.001)$ and on institutionalized patients at admission $(p<0.001)$, but no differences on KI were achieved regarding new institutionalized patients at discharge, gender of patients or the early surgery rates $(<24 h / 48 h, p>0.05$, in all cases).

The distributions of KI punctuation regarding the 30-day mortality of patients are shown in Table 2 and represented in Figure 2. KI was significantly related to 30-day mortality BI was significantly related to 30day mortality (OR per point $=1.254$ [1.089-1.444], $p=0.002$ ).

The inflection point previously determined for KI (KIIP) was set at B category, grouping patients displaying high independence (KI A-B) and patients showing any dependency (KI C-G). Analyzing the study 
population by the distribution of KIIP, we observe no differences regarding the gender of patients or new institutionalized patients at discharge $(p>0.05)$, but a lower independent patient $(K I A-B)$ rate on the institutionalized population at admission $(p<0.001)$ and aging patients $(p<0.001)$. We noted a greater proportion of independent patients operated within the first $48 \mathrm{~h}(p=0.018)$. We noted a significantly decreased risk for early mortality at this point, noting half the risk for high independent patients $\left(\mathrm{OR}_{\mathrm{KI}(\mathrm{A}-}\right.$ B) $=0.493[0.273-0.891], p=0.019)$.

\section{Lawton-Brody Index}

The mean punctuation in the LBI of our population was $3.07 \pm 2.74$. We noted significant increasing LBI punctuations on men, on aging groups and institutionalized patients at admission ( $p<0.001$, in all cases), but no differences on LBI were achieved regarding new institutionalized patients at discharge or the early surgery rates $(<24 \mathrm{~h} / 48 \mathrm{~h}, \mathrm{p}>0.05$ in all cases).

In Table 3 are shown the distributions of LBI punctuation regarding the 30-day mortality of patients, represented in Figure 2. We showed that LBI distribution was similar in survival and early mortality groups ( $p>0.05$ ), but the single LBI punctuation was significantly related to 30 -day mortality (OR per point $=0.885$ [0.788-0.992], $p=0.037)$.

The inflection point previously determined for LBI (LBIIP) was set at 4, distinguishing patients scored 3 or less and patients scored 4 or more. Analyzing the study population by the distribution of LBIIP, we observe differences regarding the gender of patients $(p=0.006)$, aging patients $(p<0.001)$, and the institutionalized population at admission $(p<0.001)$, but no for new institutionalized patients at discharge $(p>0.05)$. We noted a greater proportion of patients scored 4-8 operated within the first $48 \mathrm{~h}(p=0.004)$. We noted a tendency for early mortality at this point, but no statistical significance was achieved $\left(\mathrm{OR}_{\mathrm{LB}(0-}\right.$ $3)=1.801$ [0.932-3.480], $p=0.080$ ).

Physical Red Cross Scale

The mean punctuation in PRCS of our population was $2.0 \pm 0.93$. We noted significant increasing PRCS on aging groups $(p<0.001)$ and on institutionalized patients at admission $(p<0.001)$, but no differences on PRCS were achieved regarding the gender of patients, among hospital-derived institutionalized patients or time to surgery $(<24 \mathrm{~h} / 48 \mathrm{~h}, \mathrm{p}>0.05$, in all cases).

In Table 3 are shown the distributions of PRCS punctuation regarding the 30-day mortality of patients. We showed that PCRS distribution was similar in survival and early mortality groups (Figure 2, p>0.05), but the single PRCS punctuation was significantly related to an increased 30-day mortality rate (Figure 1, OR per point $=1.483$ [1.094-2.011], $\mathrm{p}=0.011$ ).

\section{Discussion}


Our study proposes the use of BI, KI, LBI and PCRS geriatric scores as stand-alone indicators of 30-day mortality after hip fracture surgery, validating the established inflection points as key factors for the hip fracture patients' management. It all allows us to obtain more detailed knowledge about the prognosis of these patients.

Hip fracture in older patients is indeed a highly demanding health problem. Nowadays, the management of older hip fracture patients has significantly improved thanks to the implementation of the Orthogeriatric Units (13-15). It leads into the CGA which grants a better understanding of the functional and clinical situation of each patient and enables the application of the appropriate therapeutic measures to each case $(1,2)$. The geriatric scores applied in the CGA are a very useful tool in the assessment of patients admitted to Orthogeriatric Units $(6,16)$. The unveiling of the functional status of patients facilitates, inter alia, the comparison of patient's management and care results.

The 30-day mortality may be an indirect indicator of the quality of care and assistance provided to older patients with hip fracture. Several studies are trying to find out the risk factors related to a worse outcome so that the recognition of these risk factors ensures the availability of health care resources for the patients who need them most. Examples of risk factors related to early mortality include age (17), gender (18), comorbidity (19), or even the waiting time to surgery $(20,21)$. Further, different multifactorial models have been proposed for the prediction of 30-day mortality, although due to the complexity of older hip fracture patients, it is very challenging to establish a perfect predictive model (5). It would be very useful to have a single multifunctional tool that, in addition to reporting on the functional status of patients, allows us to advance the prognosis of patients in the short term.

Weak information is available relating functional status to 30 -day mortality (22), although other studies have shown a relationship with in-hospital mortality $(1,6)$ and long-term mortality $(7,23-25)$. The functional status is usually evaluated through the application of geriatric scores that show the ability of patients for personal development in daily life, noting the $A D L$, IADL, or mobility.

Our population showed slightly higher mean age and slightly lower 30-day mortality rate than other previously reported studies on hip fracture populations $(26,27)$.

We have analyzed the influence of functional status measured through geriatric scores $(\mathrm{BI}, \mathrm{KI}, \mathrm{LBI}$, and PRCS) on early mortality. To our knowledge, it is the first time that a statistically significant relationship of these four geriatric scores with 30-day mortality is described in detail. The four scores analyzed show good individual discrimination.

We showed significant correlations of each geriatric score analyzed with 30-day mortality, noting that the better the functional status, the better the prognosis. This relationship was expected, although it had never been quantified as it is now. Despite this, no statistically significant association of the scores with newly institutionalized patients at discharge emerged. These results point to the use of $\mathrm{BI}, \mathrm{KI}, \mathrm{LBI}$, and PCRS as individual predictors of 30-day mortality of older hip fracture patients. We shall not forget the 
asymmetrical distribution of $\mathrm{BI}, \mathrm{KI}, \mathrm{LBI}$, and PRCS scores concerning the oldest patients and patients who were institutionalized at admission.

Since the punctuation of each geriatric score analyzed is continuous data, in order to facilitate clinical and scientific use, we suggest the use of the inflection points of the geriatric scores to recognize which patients with diverse functional characteristics can benefit from the various levels of care provided. So, we could quantify the increasing risk of early dying regarding the ability to perform ADL before hip fracture. The BIIP $(0-55)$, or otherwise, the KIIP $(A-B)$, showed the best discriminatory ability to predict the 30-day mortality after hip fracture surgery. These results agree with the previous inflection points validation on $\mathrm{BI}$ and $\mathrm{KI}$ for long-term mortality of hip fracture patients (7).

Likewise, our study presents some limitations: firstly, the common provenance of the studied population, as it is a single-center study; secondly, the limited use of PRCS, which represents a mobility index that is widely used in Spain but not internationally; lastly, we lack other geriatric tools not applied in our center, as part of a CGA addressed in other studies (like geriatric comorbidity index).

\section{Conclusions}

The geriatric scores analyzed (BI, KI, LBI and PCRS) are single-handedly related in a statistically significant way to the 30-day mortality after hip fracture surgery. We show key inflection points for $\mathrm{BI}$ and $\mathrm{KI}$ on $\mathrm{ADL}$ that singly could assess the raising 30-day mortality risk after hip fracture surgery.

In our belief, the fact that a commonly used tool, as the geriatric scores are, in the assessment of hip fracture patients can also be used as a singly predictor of 30-day mortality makes them very useful tools. Especially, if we consider that for $\mathrm{BI}$ and $\mathrm{KI}$ we validate the inflection points from which the prognosis worsens markedly.

\section{Abbreviations}

ADL: Activities of daily living; BI:Barthel index; Cl:Confidence interval; CGA:Comprehensive geriatric assessment; FAC:Functional Ambulation Classification; IADL:Instrumental activities of daily living; IP:Inflection point; KI:Katz index; LBI:Lawton-Brody index; OR:Odds ratio; PCRS:Physical Red Cross Scale; UHS:University Hospital of Salamanca.

\section{Declarations}

\section{Ethics approval and consent to participate}

All participants (or their relatives) have given their written informed consent to participate. The whole study was conducted following the Declaration of Helsinki and previously approved by the ethics committee for clinical research (CEIm) of the University Hospital of Salamanca. 


\section{Consent for publication}

Not applicable.

\section{Availability of data and materials}

The data supporting this study are available from the corresponding author upon reasonable request.

\section{Competing interest}

The authors have no conflicts of interest to declare.

\section{Funding}

No specific funding for this study and its publication was obtained.

\section{Authors' Contributions}

CdaC, $\mathrm{CPH}$, and AGR collected patient data and contributed to the database fill-in process. CdaC performed data analysis and contributed to draft the work. JFB designed the study and was responsible for data interpretation. All authors approved the final version of the manuscript.

\section{Acknowledgments}

Not applicable.

\section{References}

1. Avelino-silva TJ, Farfel JM, Curiati JAE, Amaral JRG, Campora F, Jacob-filho W. Comprehensive geriatric assessment predicts mortality and adverse outcomes in hospitalized older adults. BMC Geriatr. 2014;14:129.

2. Parker SG, McCue P, Phelps K, McCleod A, Arora S, Nockels K, et al. What is Comprehensive Geriatric Assessment (CGA)? An umbrella review. Ruggiero C, editor. Age Ageing. 2018 Jan 1;47(1):149-55.

3. Desforges JF, Applegate WB, Blass JP, Williams TF. Instruments for the Functional Assessment of Older Patients. N Engl J Med. 1990;322(17):1207-14.

4. Marufu TC, Mannings A, Moppett IK. Risk scoring models for predicting peri-operative morbidity and mortality in people with fragility hip fractures: Qualitative systematic review. Vol. 46, Injury. 2015. p. 2325-34.

5. Karres J, Heesakkers NA, Ultee JM, Vrouenraets BC. Predicting 30-day mortality following hip fracture surgery: Evaluation of six risk prediction models. Injury. 2015;46(2):371-7.

6. Kim K, Park K-H, Koo K-H, Han H-S, Kim C-H. Comprehensive geriatric assessment can predict postoperative morbidity and mortality in elderly patients undergoing elective surgery. Arch Gerontol Geriatr. 2013;56(3):507-12. 
7. da Casa C, Pablos-Hernández C, González-Ramírez A, Julián-Enriquez JM, Blanco JF. Geriatric scores can predict long-term survival rate after hip fracture surgery. BMC Geriatr. 2019;19(1):205.

8. Mahoney Fl, Barthel DW. Functional evaluation: The Barthel Index. Md State Med J. 1965;14:61-5.

9. Katz SM, Ford AB, Moskowitz RW, Jackson BA, Jaffe MW. Studies of Illness in the Aged: The Index of ADL. A Standardized Measure of Biological and Psychosocial Function. JAMA Jornoal Am Med Assoc. 1963;185(12):914-9.

10. Lawton MP, Brody EM. Assessment of older people: self-maintaining and instrumental activities of daily living. Gerontologist. 1969;9(3):179-86.

11. Vergara I, Bilbao A, Orive M, Garcia-Gutierrez S, Navarro G, Quintana J. Validation of the Spanish version of the Lawton IADL Scale for its application in elderly people. Health Qual Life Outcomes. 2012;10(1):130.

12. Guillén LLera F, García Antón A. Ayuda a domicilio. Aspectos médicos en geriatría. Rev Española Gerontol. 1972;7:339-46.

13. Grigoryan KV, Javedan H, Rudolph JL. Orthogeriatric Care Models and Outcomes in Hip Fracture Patients. J Orthop Trauma. 2014;28(3):e49-55.

14. Kristensen PK, Thillemann TM, Søballe K, Johnsen SP. Can improved quality of care explain the success of orthogeriatric units? A population-based cohort study. Age Ageing. 2016;45(1):66-71.

15. Pablos-Hernández C, González-Ramírez A, da Casa C, Luis MM, García-Iglesias MA, Julián-Enriquez $\mathrm{JM}$, et al. Time to Surgery Reduction in Hip Fracture Patients on an Integrated Orthogeriatric Unit: A Comparative Study of Three Healthcare Models. Orthop Surg. 2020;2:457-62.

16. Mayoral AP, Ibarz E, Gracia L, Mateo J, Herrera A. The use of Barthel index for the assessment of the functional recovery after osteoporotic hip fracture: One year follow-up. PLoS One. 2019;14(2):e0212000.

17. Forni C, Gazineo D, D'Alessandro F, Fiorani A, Morri M, Sabattini T, et al. Predictive factors for thirty day mortality in geriatric patients with hip fractures: a prospective study. Int Orthop. 2019;43(2):27581.

18. Kannegaard PN, van der Mark S, Eiken P, Abrahamsen B. Excess mortality in men compared with women following a hip fracture. National analysis of comedications, comorbidity and survival. Age Ageing. 2010;39(2):203-9.

19. Sheikh HQ, Hossain FS, Aqil A, Akinbamijo B, Mushtaq V, Kapoor H. A Comprehensive Analysis of the Causes and Predictors of 30-Day Mortality Following Hip Fracture Surgery. Clin Orthop Surg. 2017;9(1):10-8.

20. Moja L, Piatti A, Pecoraro V, Ricci C, Virgili G, Salanti G, et al. Timing Matters in Hip Fracture Surgery: Patients Operated within 48 Hours Have Better Outcomes. A Meta-Analysis and Meta-Regression of over 190,000 Patients. PLoS One. 2012;7(10):e46175.

21. Nyholm AM, Gromov K, Palm H, Brix M, Kallemose T, Troelsen A, et al. Time to Surgery Is Associated with Thirty-Day and Ninety-Day Mortality After Proximal Femoral Fracture. J Bone Jt SurgeryAmerican Vol. 2015 Aug 19;97(16):1333-9.

Page 10/16 
22. Parada Freitas Y, Fernández Arana L, Garay Airaghi V, Lueje Alonso E, Mora Fernández J. Mortalidad temprana y tardía en el paciente anciano con fractura de cadera. Rev Esp Geriatr Gerontol. 2018 Jun;53:40-1.

23. Martínez-Velilla N, Ibáñez-Beroiz B, Cambra-Contin K, Alonso-Renedo J. Is Comprehensive Geriatric Assessment a Better 1-Year Mortality Predictor Than Comorbidity and Prognostic Indices in Hospitalized Older Adults? J Am Geriatr Soc. 2013;61(10):1821-3.

24. Martínez-Velilla N, Cambra-Contin K, Ibáñez-Beroiz B. Comorbidity and prognostic indices do not improve the 5-year mortality prediction of components of comprehensive geriatric assessment in hospitalized older patients. BMC Geriatr. 2014;14(1):64.

25. Kristensen MT, Kehlet $H$. The basic mobility status upon acute hospital discharge is an independent risk factor for mortality up to 5 years after hip fracture surgery Survival rates of 444 pre-fracture ambulatory patients evaluated with the Cumulated Ambulation Score. Acta Orthop. 2018;89(1):4752.

26. Giannoulis D, Calori GM, Giannoudis PV. Thirty-day mortality after hip fractures: has anything changed? Eur J Orthop Surg Traumatol. 2016;26(4):365-70.

27. Ojeda-Thies C, Sáez-López P, Currie CT, Tarazona-Santalbina FJ, Alarcón T, Muñoz-Pascual A, et al. Spanish National Hip Fracture Registry (RNFC): analysis of its first annual report and international comparison with other established registries. Osteoporos Int. 2019;30(6):1243-54.

\section{Tables}

Table 1. Biodemographic and fracture-related features of patients. 


\begin{tabular}{|llll|}
\hline \multicolumn{2}{|l|}{ Biodemographic features } & \multicolumn{2}{l|}{ Fracture-related features } \\
\hline Gender & \multicolumn{3}{l|}{ Side of fracture } \\
\hline Female & $73.3 \%$ & Right & $47.6 \%$ \\
\hline Male & $26.7 \%$ & Left & $52.4 \%$ \\
\hline Age (mean \pm SD) & $86.27 \pm 6.78$ & Type of fracture & \\
\hline $65-79$ years-old & $16.1 \%$ & Intracapsular & $41.4 \%$ \\
\hline $80-89$ years-old & $51.3 \%$ & Basicervical & $4.6 \%$ \\
\hline$>90$ years-old & $32.6 \%$ & Trochanteric & $44.0 \%$ \\
\hline Residence & & Subtrochanteric & $6.0 \%$ \\
\hline Town & $51.6 \%$ & Periprosthetic & $4.0 \%$ \\
\hline Rural & $48.4 \%$ & Surgical procedure & \\
\hline Institution-living & & Osteosynthesis & $57.3 \%$ \\
\hline At admission & $32.0 \%$ & Hemiarthroplasty & $40.3 \%$ \\
\hline At discharge* & $54.5 \%$ & Total hip arthroplasty & $2.4 \%$ \\
\hline
\end{tabular}

Data showing absolute percentages on the study population $(n=899)$, unless otherwise specified. * Inhospital exitus are excluded from the analysis $(n=866)$. 


\begin{tabular}{|clll|}
\hline & $\begin{array}{l}\text { Survival } \\
(\mathrm{n}=846)\end{array}$ & $\begin{array}{l}30 \text {-day mortality } \\
(\mathrm{n}=53)\end{array}$ & p-value \\
\hline Barthel Index & $73.32 \pm 23.34$ & $64.62 \pm 23.82$ & 0.008 \\
\hline Grouped BI & & & 0.014 \\
\hline Independent (100) & $18.7 \%$ & $11.3 \%$ & \\
\hline Slight dependence (60-95) & $57.3 \%$ & $45.3 \%$ & \\
\hline Moderate dependence (40-55) & $15.2 \%$ & $32.1 \%$ & 0.002 \\
\hline Severe dependence (20-35) & $7.0 \%$ & $7.5 \%$ & 0.033 \\
\hline Complete dependence (<20) & $1.8 \%$ & $3.8 \%$ & \\
\hline BI Inflection Point (0-55) & $24.0 \%$ & $43.4 \%$ & 0.017 \\
\hline Katz Index & & & \\
\hline A & $32.7 \%$ & $18.9 \%$ & \\
\hline B & $16.2 \%$ & $13.2 \%$ & \\
\hline C & $18.7 \%$ & $13.2 \%$ & $17.0 \%$ \\
\hline D & $12.5 \%$ & $13.2 \%$ & \\
\hline E & $7.0 \%$ & $5.7 \%$ & \\
\hline F & $8.4 \%$ & $4.5 \%$ & \\
\hline G & $48.9 \%$ & & \\
\hline KI Inflection Point (A-B) & & & \\
\hline
\end{tabular}




\begin{tabular}{|clll|}
\hline & $\begin{array}{l}\text { Survival } \\
(\mathrm{n}=846)\end{array}$ & $\begin{array}{l}30 \text {-day mortality } \\
(\mathrm{n}=53)\end{array}$ & p-value \\
\hline Lawton-Brody Index & & & 0.546 \\
\hline 0 & $20.1 \%$ & $30.2 \%$ & \\
\hline 1 & $14.4 \%$ & $18.9 \%$ & \\
\hline 2 & $18.9 \%$ & $20.8 \%$ & \\
\hline 3 & $12.1 \%$ & $7.5 \%$ & \\
\hline 4 & $5.9 \%$ & $3.8 \%$ & \\
\hline 5 & $3.8 \%$ & $3.8 \%$ & 0.076 \\
\hline 6 & $4.1 \%$ & $1.9 \%$ & 0.141 \\
\hline 7 & $9.1 \%$ & $3.8 \%$ & \\
\hline 8 & $11.6 \%$ & $9.4 \%$ & \\
\hline LBI Inflection Point (0-3) & $65.5 \%$ & $77.4 \%$ & \\
\hline Physical Red Cross Scale & & & \\
\hline Full ambulatory capability (0) & $3.9 \%$ & $1.9 \%$ & \\
\hline Slight difficulty - no technical aids (1) & $29.2 \%$ & $18.9 \%$ & $3.0 \%$ \\
\hline One supporting point (2) & $37.0 \%$ & $34.0 \%$ & \\
\hline Two supporting points (3) & $26.2 \%$ & $37.7 \%$ & \\
\hline Wheel chair (4) & $3.7 \%$ & $7.5 \%$ & \\
\hline
\end{tabular}

\section{Figures}




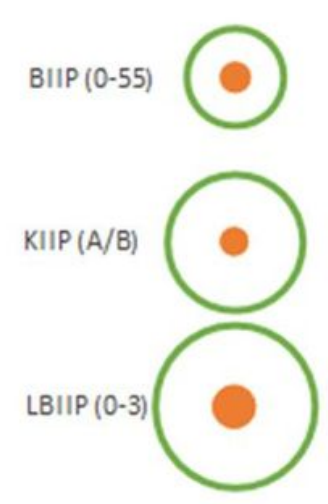

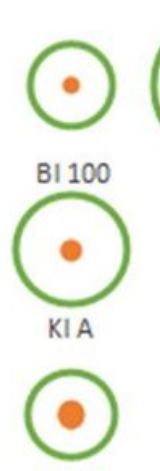

LBI 0

$\odot$

PRCS O

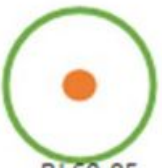

$\odot$

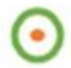

(

BI 40-55 BI $20-35 \quad$ BI $<20$

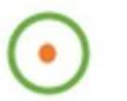

KI B

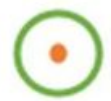

KIC

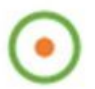

KI D

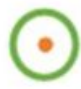

LBI 3

LBI 1

LBI 2
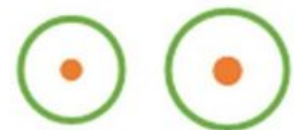

$(0)$

PRCS 3

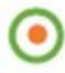

KIE

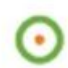

LBI 4

(อ)

PRCS 4

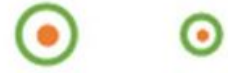

KIF KIG

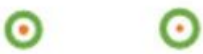

LBI 5

LBI 6

LBI 7

LBI 8

- Population on each category Early mortality rate

\section{Figure 1}

Representation of the study population on each category (ring) of the Barthel Index, Katz Index, LawtonBrody Index, and Physical Red Cross Scale. Dots are noting the early mortality rate on each category for the four geriatric scores.

A

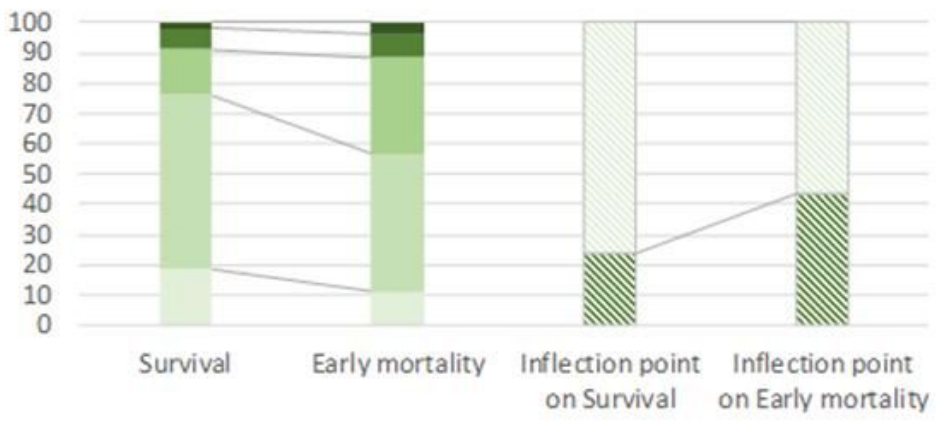

$B|100=B| 60-95=B|40-55=B| 20-35=B|<20 \leqslant B| 0-55 \square B \mid 60-100$

C

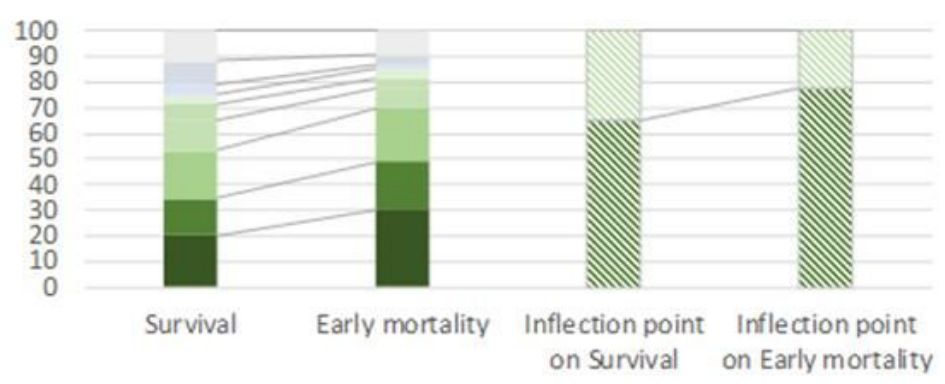

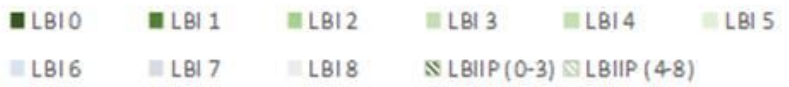

B

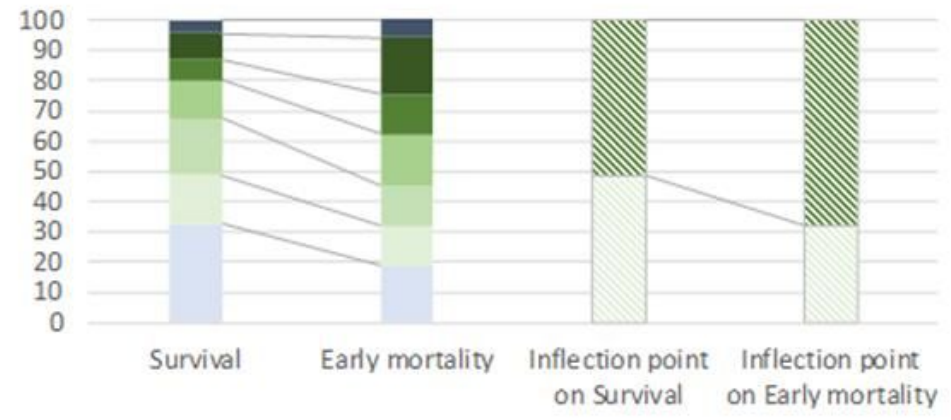

$\mathrm{KIA} \mathrm{KIB} \equiv \mathrm{KIC} \equiv \mathrm{KID}=\mathrm{KIE} \mathrm{KIF}=\mathrm{KIG} \square \mathrm{KIIPA} / \mathrm{B} \quad \mathrm{KIIPC} / \mathrm{G}$

D

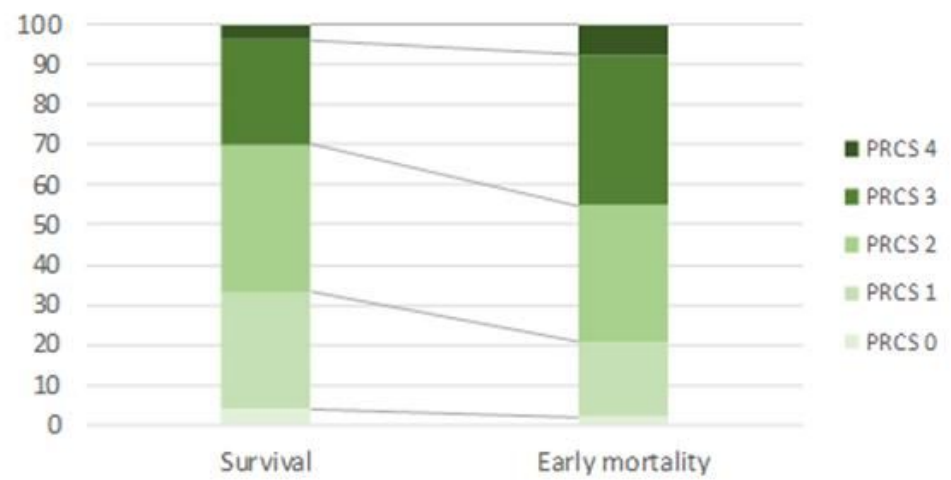

Figure 2 
Bar chart of the survival and early mortality population for Barthel Index (A), Katz Index (B), Lawton-Brody Index (C), and Physical Red Cross Scale (D). 